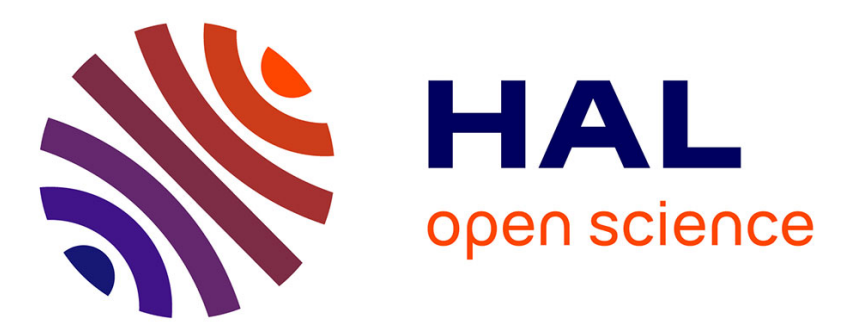

\title{
Rheology of a confined vesicle suspension
}

Abdessamad Nait-Ouhra, Alexander Farutin, Hamid Ez-Zahraouy, Abdelilah

Benyoussef, Chaouqi Misbah

\section{To cite this version:}

Abdessamad Nait-Ouhra, Alexander Farutin, Hamid Ez-Zahraouy, Abdelilah Benyoussef, Chaouqi Misbah. Rheology of a confined vesicle suspension. Physical Review Fluids, 2019, 4 (10), 10.1103/PhysRevFluids.4.103602 . hal-02397432

\section{HAL Id: hal-02397432 \\ https://hal.science/hal-02397432}

Submitted on 18 Dec 2020

HAL is a multi-disciplinary open access archive for the deposit and dissemination of scientific research documents, whether they are published or not. The documents may come from teaching and research institutions in France or abroad, or from public or private research centers.
L'archive ouverte pluridisciplinaire HAL, est destinée au dépôt et à la diffusion de documents scientifiques de niveau recherche, publiés ou non, émanant des établissements d'enseignement et de recherche français ou étrangers, des laboratoires publics ou privés. 


\title{
Rheology of a confined vesicle suspension
}

\author{
Abdessamad Nait-Ouhra, ${ }^{1,2}$ Alexander Farutin, ${ }^{1}$ Hamid \\ Ez-Zahraouy, ${ }^{2}$ Abdelilah Benyoussef, ${ }^{2,3}$ and Chaouqi Misbah ${ }^{1}$ \\ ${ }^{1}$ Univ. Grenoble Alpes, CNRS, LIPhy, F-38000 Grenoble, France \\ ${ }^{2}$ Laboratoire de Matière Condensée et Sciences Interdisciplinaires, \\ Faculty of Sciences, Mohammed V University of Rabat, 1014 Morocco \\ ${ }^{3}$ Hassan II Academy of Science and Technology, Rabat, 10220 Morocco
}

Rheology of a confined suspension of vesicles (model for blood) is analyzed numerically in two dimensions as a function of the viscosity contrast $\lambda$ (defined as the ratio between the inner and the outer viscosities $\eta_{\text {in }}$ and $\eta_{\text {out }}$ ). We consider both the dilute, semi dilute and more concentrated regimes up to about $35 \%$ of vesicle area fraction. This paper is a follow up of our recent work [A. Nait-Ouhra et al., Phys. Rev. Fluids 3, 123601 (2018).] where we have found that depending on $\lambda$ the vesicle can either be centered or off-centered in a channel. Here we will analyze in particular how the existence of the new attractor (off-centered solution) affects rheology. In the dilute regime, it is found that the effective viscosity corresponding to the off-centered solution may have a lower value than that of the centered solution. Moreover the effective viscosity shows a discontinuity as a function of $\lambda$. This discontinuity is traced back to the existence of a saddle node bifurcation of the lateral position. When the flow strength is increased the viscosity behavior becomes continuous, owing to the evolution of the transition of the off-centered solution from a saddle-node to a pitchfork bifurcation. We analyze then rheology as a function of the suspension concentration $\varphi$ for different values of $\lambda$. For large enough $\lambda$ the viscosity may be lower or larger than that corresponding to small $\lambda$, depending on initial configuration. There is a critical concentration beyond which the initial configuration is irrelevant, but still the behavior of the suspension viscosity with $\varphi$ for low and large $\lambda$ are quite different both qualitatively and quantitatively. This is traced back to special spatial organization of the confined suspensions, which depends on $\lambda$. 


\section{INTRODUCTION}

Rheology of confined suspensions, where the suspended entity size becomes of the order of the available gap, has received so far much less attention than their unconfined counterparts. There are many examples where the confinement effects come to the fore. For example, in microfluidics and nanofluidics [1-11] the transported entities (cells, macromolecules, such as DNA, and so on) have often sizes which can be comparable to the typical size of the transport devices. We therefore expect the interaction of the suspended entities with walls to play decisive roles. For example, walls may create depletion (zones), due either to entropic or hydrodynamic effects [10, 12-17] that should affect transport properties. A prominent example is blood flow where red blood cells (RBCs) interaction with the vessel walls leads to a cell-free layer close to the wall. This cell-free layer plays a pivotal role in oxygen transport, since it leads to a drastic reduction of the effective viscosity with decreasing vessel diameter, allowing thus for a more efficient transport in small vessels of the vascular network where oxygen (and other elements, such as ATP[18]) is delivered to tissues and organs. The decrease of blood viscosity with vessel diameter is known as the FåhræusLindqvist effect [19]. A basic understanding of the behavior of confined suspensions requires, on the one hand, the identification of the key parameters that play a relevant role, and on the other hand linking the microscale (e.g. the spatiotemporal organization) to the macroscale. The present study is directed along this objective. We will see that the viscosity contrast $\lambda$ (defined as the ratio between the inner viscosity $\eta_{\text {in }}$ and the outer one $\eta_{\text {out }}$ ) plays an essential role in the spatial organization, which in turn affects rheology. The rheology of confined suspensions has been addressed quite sparsely to date. Regarding suspensions of rigid spheres, Peyla et al. [20-22] have shown that for a certain range of ratio between the gap size and the particle size, the interactions between particles leads to a decrease of the total dissipation. 2D and 3D numerical simulations of confined RBCs [23, 24] have been performed using vesicles and capsules as a model. It has been shown that confinement leads to a subtle ordering with a strong impact on rheology. Similar ordering [25] has been reported on confined suspensions of rigid paricles in the presence of inertia.

Our study is motivated by blood flow. However, since our objective is to analyze the basic fundamental features, we focus here on a simplified model: a 2D suspension of vesicles (a vesicle is a closed phospholipid membrane [26]). It has been shown that $2 \mathrm{D}$ vesicles capture many features known for 3D models of RBCs. Indeed, both models show several common shapes and dynamics, namely: tank-treading and tumbling [27, 28], slipper and parachute shapes $[29,30]$, flow alignment $[27,31]$ and very recently polylobes shapes $[32,33]$ etc. In addition, both models show the same rheological behavior [23]. These observations provide confidence that the adopted $2 \mathrm{D}$ system may retain the essential effects of a more realistic model, and at the same time offers low enough numerical cost for a more systematic investigation.

An important factor in our study is the role of the viscosity contrast $\lambda$. It is traditionally reported that under shear flow (in 2D) two basic dynamics prevail: tank-treading (TT, obtained for low $\lambda$ ) and tumbilng (TB, obtained for large $\lambda$ ). In the presence of confining walls, a vesicle under shear flow (obtained by two countertranslating plates) is known to migrate towards the centerline, be it in the TT or TB regime (in the TB regime the migration velocity is smaller than in the TT one) [31, 34]. The migration due to a wall has been the subject of many studies [34-42].

Recently [31], we have discovered that a vesicle under a confined linear shear flow in the Stokes limit has other final solutions than settling in the centerline. More precisely, for a small enough flow strength and beyond a certain $\lambda$ the central position coexists with an off-centered one. The off-centered solution arises as a saddle-node bifurcation. When the flow strength increases, the saddle node bifurcation transforms into a pitchfork bifurcation. Here we study how the emergence of the new attractors affects rheology. We shall see that the new solutions significantly impact the rheology, provided that the suspension concentration is small enough. When the concentration exceeds a certain value, we shall see that the coexistence of attractors in the dilute regime is overridden by hydrodynamic interactions. However, the viscosity contrast still plays an important role by changing qualitatively and quantitatively the rheological properties.

\section{MODEL AND METHOD}

Here we describe briefly the membrane model and the simulation method (for further information see [31, 43]). We consider a set of $2 \mathrm{D}$ vesicles inside a channel, under a planar shear flow $\left(\mathbf{v}^{0}=\dot{\gamma} y \mathbf{e}_{x}\right)$. The vesicle membrane influences the ambient fluid via bending and tension forces, and the total membrane force is given by:

$$
\mathbf{f}_{\mathrm{mem} \rightarrow \mathrm{flu}}=\kappa\left(\frac{d^{2} c}{d s^{2}}+\frac{1}{2} c^{3}\right) \mathbf{n}-\zeta c \mathbf{n}+\frac{d \zeta}{d s} \mathbf{t}
$$

which includes the local effective tension term $\zeta$ in order to fulfill the local arclength conservation constraint $(\zeta$ is a Lagrange multiplier). Here $c$ is the local curvature, $s$ is the curvilinear coordinate along the membrane, $\mathbf{n}$ and $\mathbf{t}$ 
are the unit normal and tangent vectors, and $\kappa$ is the membrane bending rigidity. The enclosed area $A$ is conserved automatically thanks to the fluid incompressibility. However, very slight variations of area and perimeter are observed because of numerical errors (see Fig.1 in [44]). A derivation of Eq.(1) can be found in [42], obtained from the functional derivative of the Helfrich [45] bending energy $E=\frac{\kappa}{2} \int_{\text {mem }} c^{2} d s+\int_{\text {mem }} \zeta d s$.

We consider the limit of zero Reynolds number (the Stokes limit). The full problem is described by four dimensionless numbers, which are:

$$
\lambda=\frac{\eta_{\text {in }}}{\eta_{\text {out }}}, C_{\kappa}=\frac{\eta_{\text {out }} \dot{\gamma} R_{0}^{3}}{\kappa} \equiv \dot{\gamma} t_{c}, C_{n}=\frac{2 R_{0}}{W}, \tau=\frac{4 \pi A}{L^{2}}
$$

$\lambda$ is viscosity contrast, where $\eta_{i n}, \eta_{\text {out }}$ denote the inner and the outer viscosities. $C_{\kappa}$ is the capillary number measuring the flow strength over the bending energy of the membrane. In other words, $C_{\kappa}$ controls how the shapes of vesicles deform in response to an applied external flow, where $t_{c}$ is the typical time needed for the vesicle to recover its equilibrium shape after cessation of flow. $\dot{\gamma}$ is the applied shear rate. $C_{n}$ is the degree of confinement, where $W$ is the channel width. $\tau$ is the reduced area, where $L$ is the vesicle perimeter, the effective vesicle radius is defined as $R_{0}=\sqrt{A / \pi}$. Using the Green's function techniques, the velocity $\mathbf{v}\left(\mathbf{r}_{0}\right)$ of a point on the membrane can be written in the following dimensionless expression:

$$
\mathbf{v}\left(\mathbf{r}_{0}\right)=\frac{2}{1+\lambda} \mathbf{v}^{0}\left(\mathbf{r}_{0}\right)+\frac{1}{2 \pi C_{\kappa}(1+\lambda)} \int_{\text {mem }} d s \mathbf{f}_{\mathrm{mem} \rightarrow \mathrm{flu}}(\mathbf{r}) \cdot \mathrm{G}^{2 w}\left(\mathbf{r}, \mathbf{r}_{0}\right)+\frac{1-\lambda}{2 \pi(1+\lambda)} \int_{\mathrm{mem}} d s \mathbf{v}(\mathbf{r}) \cdot \mathrm{T}^{2 w}\left(\mathbf{r}, \mathbf{r}_{0}\right) \cdot \mathbf{n}(\mathbf{r})
$$

where we have used the following scales: $R_{0}$ for the length, $U=\dot{\gamma} R_{0}$ for the velocity and $\kappa / R_{0}^{3}$ for the force per unit area. $\mathrm{G}^{2 w}\left(\mathbf{r}, \mathbf{r}_{0}\right)$ and $\mathrm{T}^{2 w}\left(\mathbf{r}, \mathbf{r}_{0}\right)$ are the Green's functions (second- and third-order tensors) satisfying the no-slip boundary condition at the bounding walls (see [43] for more details). $\mathbf{v}^{0}$ is the imposed velocity.

The dimensionless force $\mathbf{f}_{\text {mem } \rightarrow \text { flu }}$ is given formally by the same expression in Eq.(1) in which membrane bending rigidity $\kappa$ is set to unity (we keep the same notation for simplicity).

The still unknown Lagrange multiplier $\zeta$ is obtained by imposing a divergence-free velocity along the membrane, expressing the membrane incompressibility. The numerical method of imposing membrane incompressibility follows closely that presented in [28].

The membrane displacement in time is obtained by updating the discretization points after each time iteration, using a Euler scheme, $\mathbf{r}_{0}(t+d t)=\mathbf{v}(t) d t+\mathbf{r}_{0}(t)$.

\section{RESULTS}

\section{A. Rheology and its relation to attractors of vesicle positions in the channel}

In a recent work [31] we have seen that the vesicle final lateral position in the channel (denoted as $\left.\mathrm{h}_{\mathrm{f}}\right)$ depends on $\lambda$. We have found that beyond a critical $\lambda$ (and for a small enough $C_{\kappa}$ ) there is emergence of a non centered solution coexisting with the centered one. In this section, we consider the very dilute regime (where the additivity effect due to each vesicle is legitimate) in order to study the impact of the lateral position on the rheology. The effective viscosity is given by:

$$
\eta_{\text {eff }}=\eta_{\text {out }}(1+[\eta] \varphi)
$$

Where $[\eta]$ is the normalized viscosity (is called the intrinsic viscosity when $\varphi \rightarrow 0$ ), represents the contribution of the vesicles to the viscosity. It is given by

$$
[\eta]=\frac{1}{\eta_{\text {out }} S \dot{\gamma}}\left[\int_{\mathrm{mem}} d s y f_{\mathrm{flu} \rightarrow \mathrm{mem}, x}+\eta_{\text {out }}(\lambda-1) \int_{\mathrm{mem}} d s\left(n_{x} v_{y}+n_{y} v_{x}\right)\right]
$$

where $S$ is the total area of the vesicles. This expression is the extension of the Batchelor result for suspensions of rigid particles to vesicles [46].

For a small enough $\lambda$ the vesicle always reaches the centerline regardless of initial position [31]. Once the final position is reached the normalized viscosity is extracted from (5). Figure 1 shows the result. Here parameters are: $\tau=0.7, C_{\kappa}=1$ and $C_{n}=0.4$. Below a critical value of $\lambda\left(\lambda_{c} \simeq 16\right.$, a value which depends on the other parameters) the vesicle migrates towards the center of the channel. In this case (centered vesicle), the suspension shows a classical behavior $[28,43,47-51]$ : in the tank-treading motion (TT) $[\eta]$ decreases with $\lambda$ until a minimal value. Then $[\eta]$ 

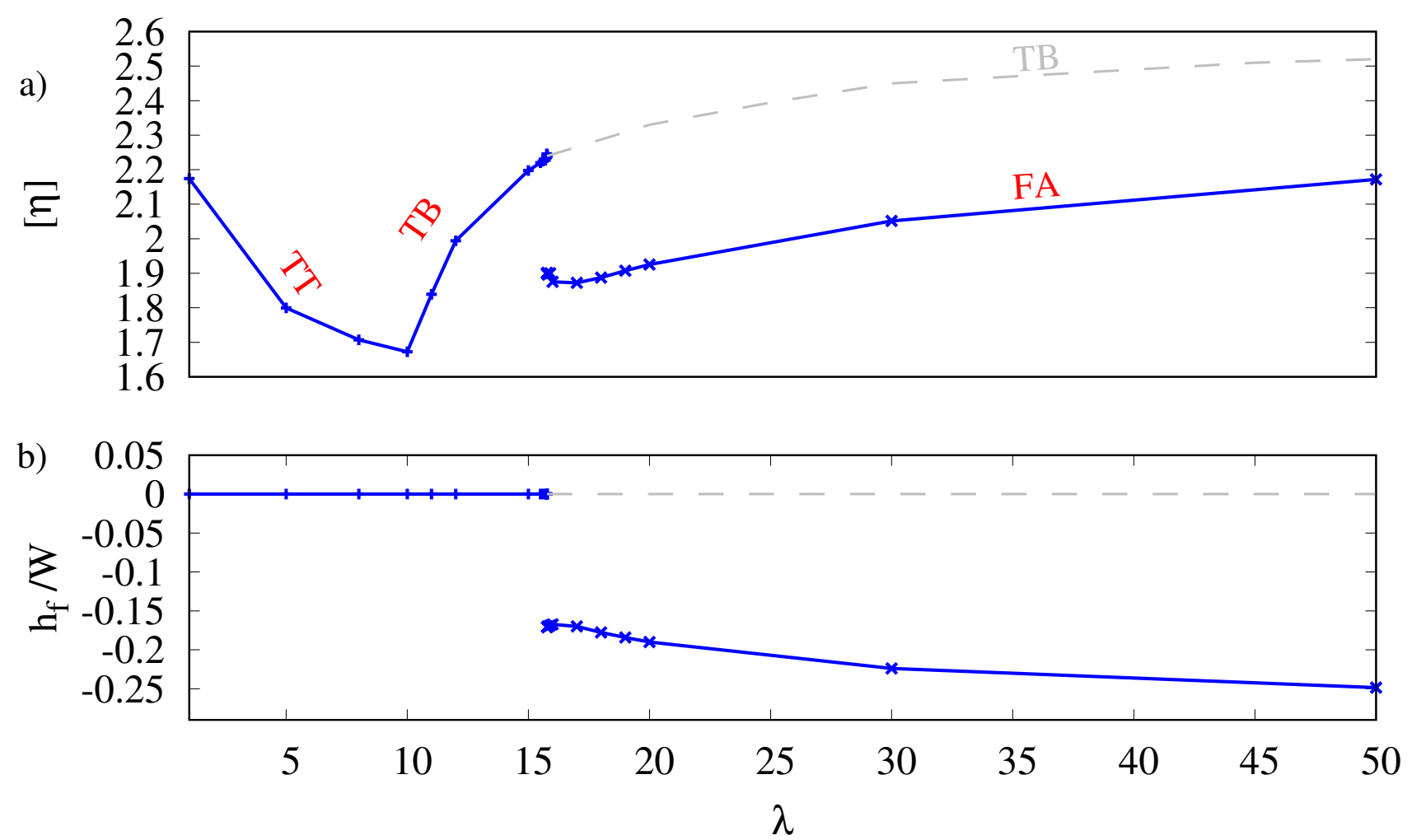

FIG. 1. (Color online) The evolution of the normalized viscosity $[\eta]$ and equilibrium lateral position $\mathrm{h}_{\mathrm{f}}(\mathrm{a}$ and $\mathrm{b} r e s p$.) as a function of the bifurcation parameter $\lambda$. TT: Tank-treading, TB: Tumbling, FA: Flow alignment. Here $\tau=0.7, C_{\kappa}=1$ and $C_{n}=0.4$. The dashed gray lines correspond to the results where the vesicle initially started at the centerline or at its vicinity and ultimately gets centered. The centerline is at $y / W=0$ on the vertical axis in (Fig. 1b) and the lower wall is at $y / W=-1 / 2$.

increases in the tumbling regime (TB) (Fig. 1a). Above $\lambda_{c}$, if the initial position is close enough to the wall (the distance from the lower wall in the simulation is $0.72 R_{0}$ ), the vesicle settles in an off-center position [31] as shown in Fig. 1b. This solution coexists with the centered solution. The vesicle aligns with the flow and we have referred to this solution as flow alignment (FA), whereas in the coexisting centered regime the vesicle shows TB. The two corresponding branches are labeled FA and TB in Fig. 1a. In other words, the suspension presents two possible values of viscosity due to the existence of two different attractors (see Fig.2 in [44] for an averaged viscosity over all possible final configurations). Whether the system selects one solution or the other depends on initial conditions. The viscosity in the FA regime is significantly smaller than that in the TB regime. This is quite intuitive since a TB vesicle has a larger cross section in the channel than a FA vesicle, opposing thus higher resistance to the flow. When $\lambda$ increases further the viscosity of the FA solution shows an increase of the suspension viscosity. This is due to the fact that the FA vesicle is closer and closer to the wall, and thus the cell free layer becomes smaller and smaller. This is reminiscent of the Fåhræus-Lindqvist effect [19].

\section{B. Effect of degree of confinement}

In this section we investigate the effect of the degree of confinement on the dynamics and rheology of a very dilute suspension of vesicles. We take a single vesicle initially placed close to the lower wall. We set $\tau=0.7, C_{\kappa}=1$ and vary $\lambda$ (key parameter in this study) for several $C_{n}$. The results are depicted in Fig. 2 which shows the same qualitative behavior as seen in the previous section. The same dynamics of the vesicle for $C_{n}=0.4$ (previous section) persists for all values of confinement investigated here, that is, TT, TB and FA motion are observed. For each value of $C_{n}$ (Fig. 2) there is a different critical viscosity contrast $\lambda_{c}$, below which the vesicle migrates to the centerline and $[\eta]$ shows a non-monotonic behavior as already discussed in the previous section. Beyond $\lambda_{c}$ the vesicle settles at an off-center position, exhibiting a sudden decrease of the normalized viscosity $[\eta]$ of the suspension (Fig. 2), which then increases with increasing $\lambda$. In conclusion, the same trend is observed pointing to the robustness of the phenomenon. 

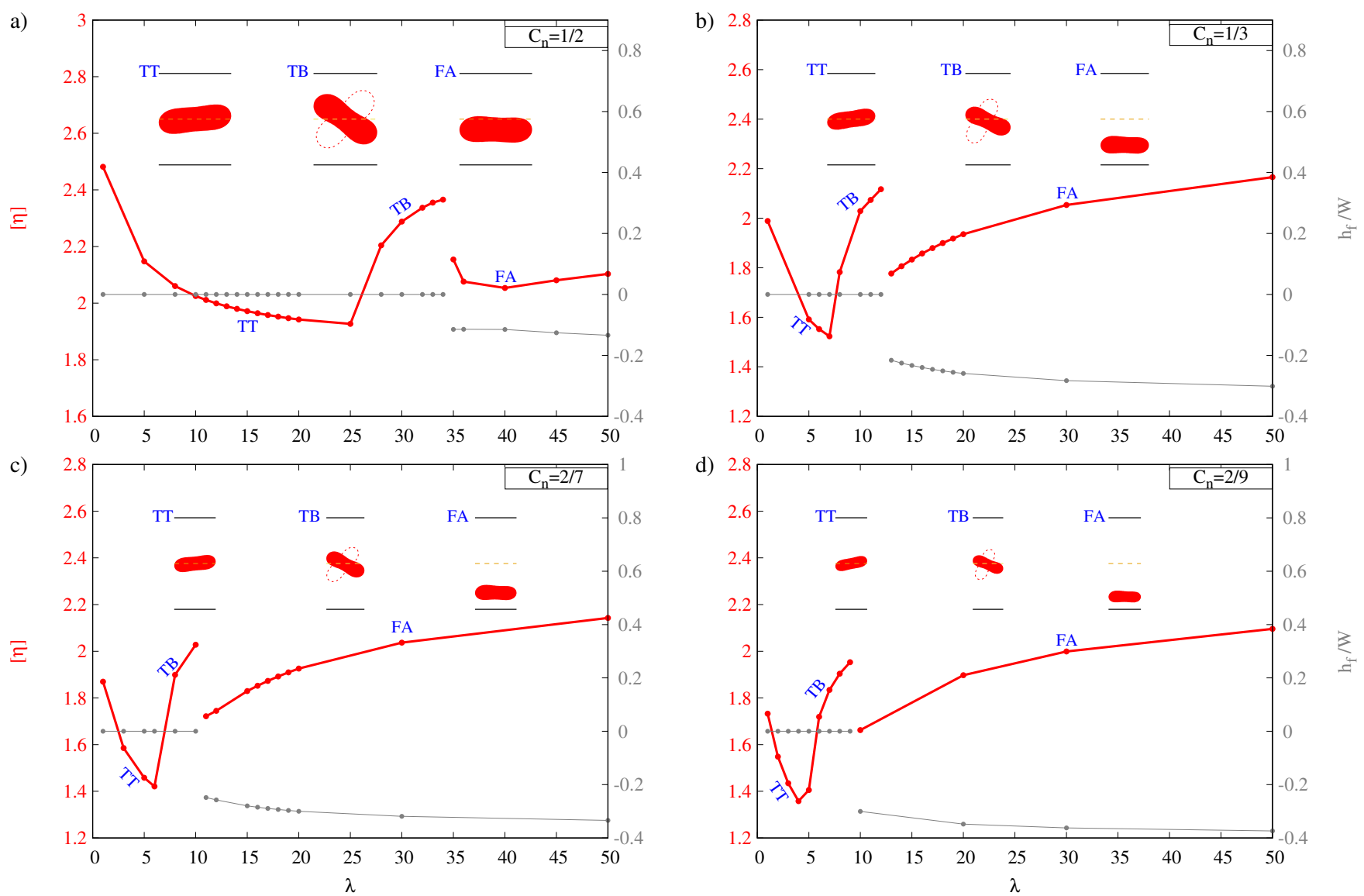

FIG. 2. (Color online) The evolution of $[\eta]$ and equilibrium lateral position $\mathrm{h}_{\mathrm{f}}$ as a function of $\lambda$ for several degrees of confinement $C_{n}$. Here we set $C_{\kappa}=1$ and $\tau=0.7$. The vesicle is initially set at a small enough distance $\left(0.72 R_{0}\right)$ from the lower wall. TT, TB and FA are shown. The channel center is at zero and the walls are at $y / W= \pm 1 / 2$ on the right vertical axis. Note that the TB branch continues for larger $\lambda$ but here we only show the results until the FA branches appears.

\section{Effect of capillary number}

In this section we consider the effect of capillary number $C_{\kappa}$ on the dynamics and rheology of the vesicle by fixing the other parameters, $C_{n}=0.4, \tau=0.7$. Figure 3 illustrates the effect of $C_{\kappa}$ on the dynamics and rheology. The main difference with previous results is that upon increasing $C_{\kappa}$ the gray line representing the off-centered position as a function of $\lambda$ exhibits weaker and weaker jumps at the bifurcation point, until the transition becomes continuous. The saddle-node bifurcation transforms into a pitchfork bifurcation. This transition also affects the behavior of the normalized viscosity $[\eta]$ which shows weaker jumps until showing a continuous behavior. A point worth of mention is that the TB regime completely disappears in favor of a continuous transition from a TT solution to a FA one. We also note that for a given $\lambda$ in the off-centered case (FA regime in Fig. 3), the distance between the vesicle and the wall increases as a function of capillary number $C_{\kappa}$ implying a decrease of the normalized viscosity with $C_{\kappa}$ (shear-thinning behavior).

\section{Effect of reduced area}

We carried out a systematic study on the effect of reduced area $\tau$ (for a circle $\tau=1$, otherwise $\tau<1$ ) on the equilbrium lateral position of a vesicle and its effect on the rheology of the suspension. The results are reported in Fig.4. The other parameters are fixed to $C_{n}=0.4$ and $C_{\kappa}=1$. When $\tau$ is small enough, we find the same trends as before. However, as $\tau$ increases (approaching 1), the situation changes significantly. For example, for $\tau=0.9$ while the vesicle migrates out of center (for $\lambda>40$ ), the normalized viscosity still exhibits a jump. Contrary to the other values of $\tau$, the viscosity is higher for the off-centered solution at the transition point. This is due to two effects: (i) the higher value of $\tau$ lowers the lift due to wall so that the fluid left between the vesicle and the wall is smaller, leading 

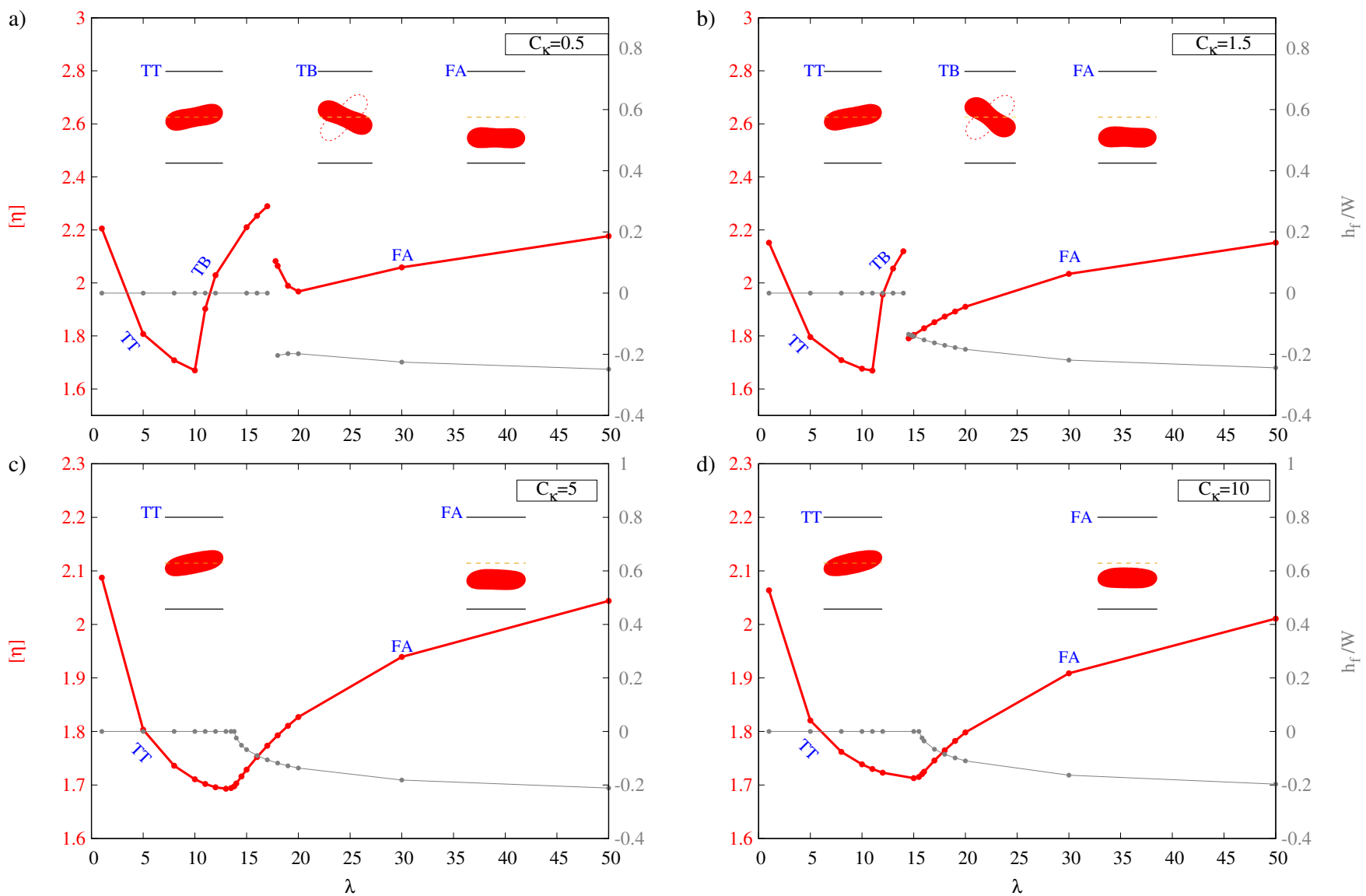

FIG. 3. (Color online) The evolution of $[\eta]$ and equilibrium lateral position $\mathrm{h}_{\mathrm{f}}$ according the bifurcation parameter $\lambda$ for several capillary numbers $C_{\kappa}$. The vesicle is initially set at a small enough distance $\left(0.72 R_{0}\right)$ from the lower wall. TT, TB and FA are shown. Beyond a certain value of $C_{\kappa}$ (c and d panels), the TB motion is suppressed and the evolution of $[\eta]$ as a function of $\lambda$ does no longer exhibit any jump. The channel center is at zero and the walls are at $y / W= \pm 1 / 2$ on the right $y$ vertical axis. Note that the TB branch (when it exists) continues for larger $\lambda$ but here we only show the results until the FA branches appears. For the continuous curves, TB regime does not exist.

to higher dissipation. (ii) The vesicle has a shape close to a circle causing a higher cross-section against the flow. These two effects together yield higher viscosity than a tumbling vesicle at the center. Even if the vesicle tumbles at the center, due to its quasi-circular shape, its elongation is weaker now causing less and less resistance against the flow.

\section{SUSPENSIONS}

We have seen above that depending on $\lambda$ a single vesicle may migrate to the center or towards the wall and this significantly alters the rheology of the suspension. The question naturally arises of whether this off-centered position may survive for higher concentrations. We have investigated this question by varying concentration and by choosing various initial vesicle positions with the aim to see whether or not the final configuration is sensitive to initial conditions. Periodic boundaries are used along $x$ direction, the box length is $L_{x}=40.5 R_{0}$ and the enclosed area of a single vesicle is $A=\pi R_{0}^{2}$. The other parameters are kept to $\lambda=20, C_{n}=0.4$ and $C_{\kappa}=1$ for which a single vesicle would select an off-centered position. We first discuss the dilute and the semi-dilute regimes. We compare the results to those obtained in Ref.[23] for another set of parameters $\left(\lambda=1, C_{n}=0.4\right.$ and $\left.C_{\kappa}=1\right)$ where only the centered solution exists; i.e. for this viscosity contrast $\lambda=1$, there exists no second attractor (off-centered) for a single vesicle.

The results are presented in Fig.5. We see that for $\lambda=20$, and below a certain concentration $(\varphi \simeq 12.4 \%)$, the normalized viscosity $[\eta]$ may have several values for the same concentration $(\varphi)$ depending on the final configuration (which depends on the initial configuration) of the suspension. This means that for dilute and semi-dilute suspensions, several stable spatial configurations are possible which give rise to different values of normalized viscosity $[\eta]$ or 

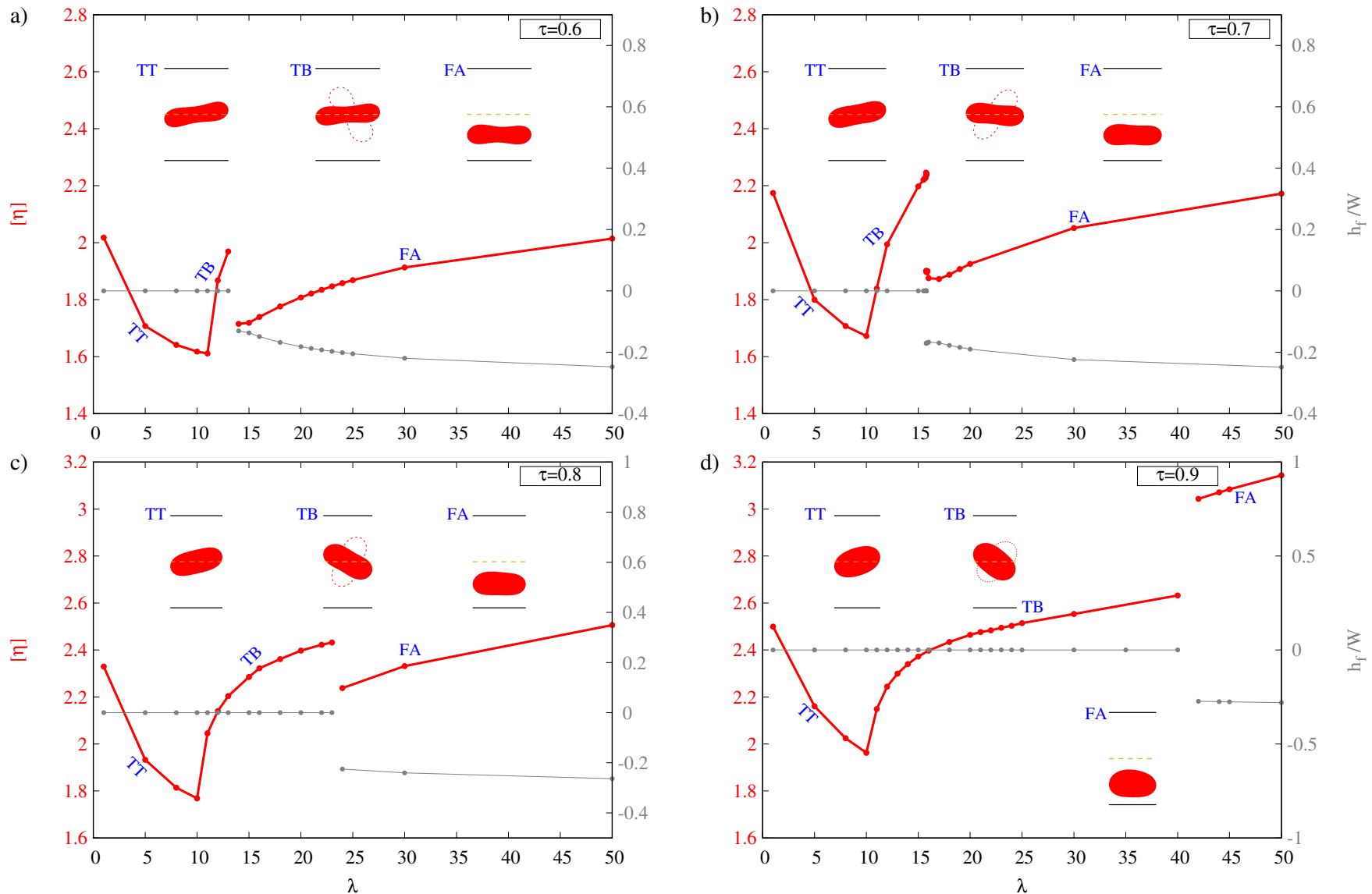

FIG. 4. (Color online) The evolution of $[\eta]$ and equilibrium lateral position $\mathrm{h}_{\mathrm{f}}$ as a function of the bifurcation parameter $\lambda$ for several reduced areas $\tau$. Here we set $C_{\kappa}=1$ and $C_{n}=0.4$. The vesicle is initially set at a small enough distance $\left(0.72 R_{0}\right)$ from the lower wall. TT, TB and FA are shown. The channel center is at zero and the walls are at $y / W= \pm 1 / 2$ on the right $y$ vertical axis. The normalized viscosity $[\eta]$ shows in the FA regime a jump with higher values than those of the TB regime for $\tau=0.9$. This contrasts with the behavior for other value of $\tau$, see text. Note that the TB branch continues for larger $\lambda$ but here we only show the results until the FA branches appears.

effective viscosity $\eta_{\text {eff }}$ (Fig.6). It is interesting to note that, below a certain concentration $(\varphi \simeq 6.2 \%)$, the suspension of relatively rigid vesicles $(\lambda=20)$ may have a much lower value of $[\eta]$ than that of a suspension of soft vesicles $(\lambda=1$ Fig.5). This lower value for more rigid particles is associated with the existence of stable off-centered positions, as described above. The highly non-monotonic behavior of $[\eta]$ for $\lambda=1$, described in [23], as a function of $\varphi$ is due to the existence of ordered solutions with a single (for low enough $\varphi$ ) and double (for larger $\varphi$ ) file configurations.

For a large enough $\lambda$ for which two attractors exist for a single vesicle, the situation is quite different. We have fixed $\lambda=20$. We find, depending on initial conditions (as described in the caption of Fig.5) that for a small enough $\varphi(<6.2 \%)$ there exist two solutions (i) centered single file (as found for $\lambda=1$ ) undergoing tumbling giving rise to the viscosity values shown as points $\left(a^{\prime}, b^{\prime}\right.$ and $\left.c^{\prime}\right)$ in Fig.5 and (ii) off-centered single file solution exhibiting flow alignment having the viscosity shown as points $(a, b$ and $c)$ in Fig.5. In other words, for low enough $\varphi$ the single ordered file seems to exists whatever the value of $\lambda$ is. For $\lambda=20$ the existence of FA single file exists only for a low enough concentration (points $a, b$ and $c$ in Fig.5). In this low concentration regime the normalized viscosity shows a plateau owing to the weak hydrodynamic interaction.

However, when the concentration increases slightly beyond point $c$ in Fig.5 the hydrodynamic interaction among vesicles starts to play a role, but still the existence of two attractors continues to affect rheology up to about $\varphi=12 \%$. The situation becomes more complex than for a lower concentration. Indeed, the single file FA solution undergoes a transition towards the centered TB file (point $d$ and inset $d$ in Fig.5). From point $c$ (FA file) to point $d$ (TB centered solution, see inset $d$ ) the viscosity increases as expected, since TB vesicles in the centerline have a higher cross section. When the concentration is increased further (from point $d$ to $e$ ) the single file TB solution still exists, but $[\eta]$ decreases. The reason is that the insertion of a new vesicles fills the gap between already existing vesicles and reduces the strength of recirculation zones between vesicles. This reasoning is similar to that evoked for $\lambda=1$ (single 
file TT solution)[23]. For solutions $d$ and $e$ the initial condition corresponds to a centered set of solution. It seems thus that the central attractor is robust despite the presence of hydrodynamic interaction. This is, however, not the case for the the second (off-centered) attractor. We have prepared half of vesicles with the off-centered position (at the attractor position for a single vesicle) and the other half at the opposite position (which is also an attractor as well) with the hope of obtaining a double file solution (as with $\lambda=1$ [23]). All simulations with these initial conditions led to a disordered final solution (represented by inset $f$ in Fig.5). The same outcome is obtained with random initial position. It seems thus that the second attractor has become unstable in the presence of hydrodynamic interactions.

A further increase of $\varphi$ (for $\lambda=20$ ) beyond point $e$, destroys the single file TB solution in favor of an apparently disordered solution (see inset $f$ ), leading to higher and higher viscosity. This is drastically different from the case of $\lambda=1$, where the system organizes into a double file that survives up to about $\varphi=35 \%$. At that concentration, the suspension for $\lambda=20$ shows a significantly higher $\eta_{e f f}$, which is about 1.45 times that corresponding to $\lambda=1$.

In summary, the suspension viscosity shows a rich behavior depending on the viscosity contrast $\lambda$. For small enough $\varphi$, the more rigid particles can either show lower or higher normalized viscosity depending on initial conditions. This points to a non trivial fact that data handling in experiments inherently contain statistical variances not only due to noise and natural imperfections and variety of samples, but also due to the very nature of multi-stability of solutions reported on here. This sheds a new light for future interpretations of rheology of soft suspensions. Beyond a certain concentration, the softer particles exhibit order (e.g. double file), whereas more rigid particles show disorder, resulting in a much higher effective viscosity. Note finally, that the appearance of order (soft enough particles) and disorder (for more rigid particles) is not a $2 \mathrm{D}$ property, but also a 3D property, shown both theoretically for model systems (drops, capsules, vesicles) and experimentally for red blood cells [24]. The present study can thus serve as a guide for a systematic analysis in $3 \mathrm{D}$.

\section{CONCLUSION}

A major central point of this study is that the existence of two attractors leads to a rheology which depends on initial configurations. This happens in the dilute and the semi-dilute regimes, up to about a concentration of $12 \%$. Beyond that concentration, the attractors loose their identities. Still, the viscosity contrast affects significantly the value of the effective viscosity as shown in Fig. 6. Several diseases (such as malaria) are accompanied by hardening of the RBC cytoplasm, a fact which can be mimicked by a higher internal viscosity (and thus higher viscosity contrast). This leads to a higher effective viscosity in microcirculation compromising the efficiency of oxygen transport. This study was dedicated to 2D and to a linear shear flow. An interesting extension should now be made for 3D and for a pipe flow which is a more relevant scenario for blood flow. Ordering of RBCs (and its impact on rheology), similar to the reported result in 2D has already been identified in 3D [23, 24]. This fact provides some confidence to the present study for its relevance to a more general situations.

\section{ACKNOWLEDGEMENTS}

We thank CNES (Centre National d'Etudes Spatiales), and the French-German university programme "Living Fluids" (Grant CFDA-Q1-14) for a financial support. A. N-O thanks CNRST Morocco for awarding a scholarship of excellence (Grant Ref. k 1/045). The simulations were performed on the Cactus cluster of the CIMENT infrastructure, which is supported by the Rhône-Alpes region (GRANT CPER07_13 CIRA). 


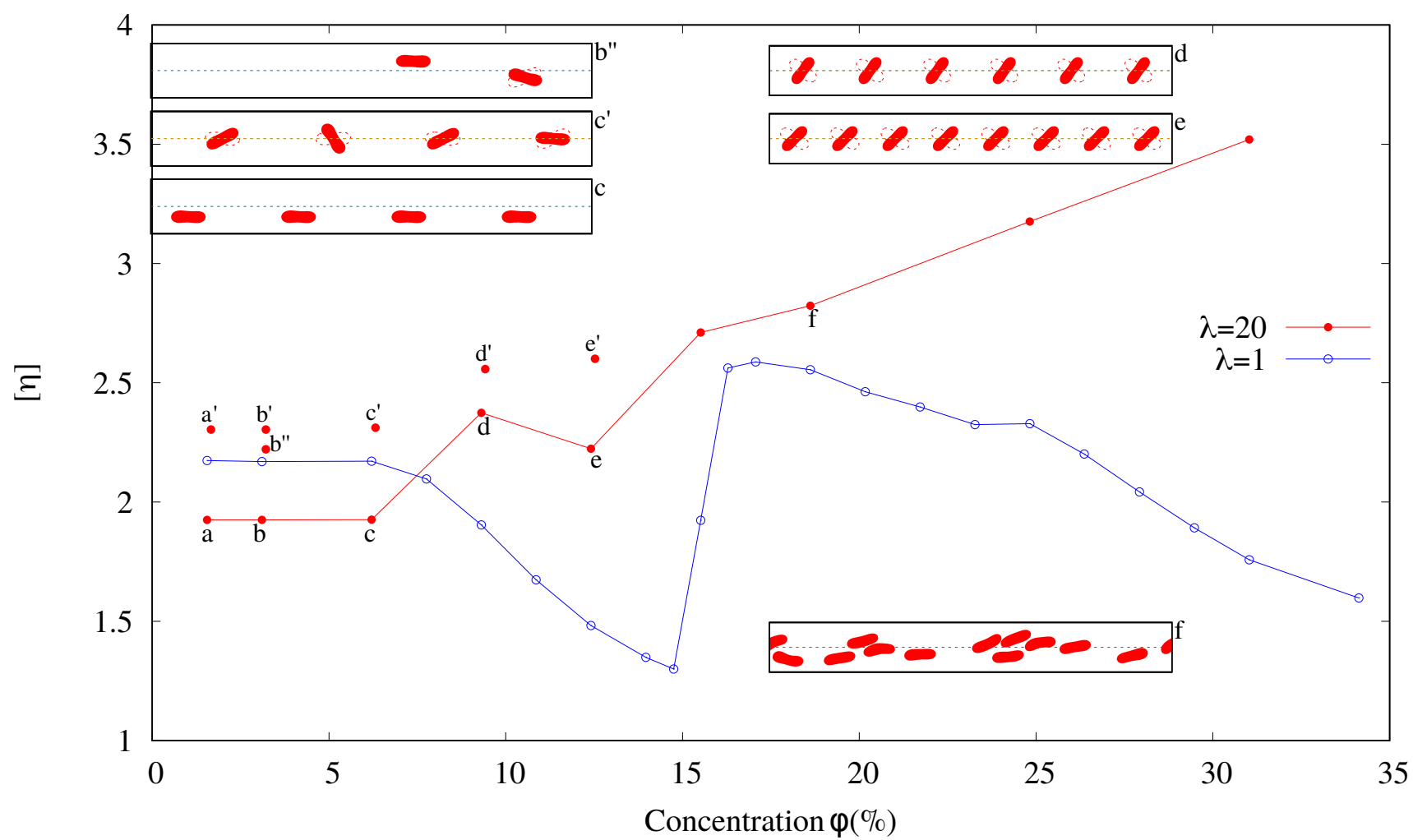

FIG. 5. (Color online) The evolution of $[\eta]$ as a function of concentration $\varphi$ for typical values of $\lambda$. Here we set $C_{\kappa}=1$ and $C_{n}=0.4$. The initial position of the vesicles has impact on the rheology up to $\varphi \simeq 12.4 \%$. Points $a, b$ and $c$ on the curve correspond to FA configuration (a typical configuration is shown in inset $c$ ). Points $a^{\prime}, b^{\prime}$ and $c^{\prime}$ on the curve correspond to another type of configuration (centered TB; a typical configuration is shown in inset $c^{\prime}$ ) obtained from different initial conditions as compared to $a, b$ and $c$ configurations. Point $b^{\prime \prime}$ on the curve corresponds to another configuration which is a mixed state, in which a vesicle undergoes an off-centered TB, whereas the second one shows FA (see inset $b^{\prime \prime}$ ). Points $d$ and $e$ on the curve correspond to centered TB configuration (see inset $d$ and $e$ resp.). Points $d^{\prime}, e^{\prime}$ and $\mathrm{f}$ on the curve correspond to disordered configuration (a typical configuration is shown in inset $f$ ). The blue curve $(\lambda=1)$ is taken from Ref.[23]. Configurations $\mathrm{a}, \mathrm{b}$ and $\mathrm{c}$ are obtained from initial conditions where the vesicle are equidistant and set close to lower wall $\left(0.72 R_{0}\right.$ distance from the wall). Configuration $d$ and $e$ are obtained with initial set of equidistant vesicles at the center. Configuration $f$ is obtained with random initial positions. Configurations $a^{\prime}, b^{\prime}$ and $c^{\prime}$ are obtained from initial conditions where the vesicle are equidistant and set at the center. It is noteworthy that we may also obtain the points $b^{\prime}$ and $c^{\prime}$ with initial random positions with a much longer time span. Configurations $d^{\prime}$ and $e^{\prime}$ are obtained with initial random positions. Configuration $b^{\prime \prime}$ is obtained with a vesicle at the center and the other close to the wall $\left(0.72 R_{0}\right.$ distance from the wall).

[1] Brian J Kirby, Micro-and nanoscale fluid mechanics: transport in microfluidic devices (Cambridge university press, 2010).

[2] Andrew N Constable and William J Brittain, "Modification of flow through silica micro-capillaries via polymer brushes," Colloids Surf. A 380, 128-134 (2011).

[3] Ai-Jun Wang, Jiu-Ju Feng, and Jing Fan, "Covalent modified hydrophilic polymer brushes onto poly (dimethylsiloxane) microchannel surface for electrophoresis separation of amino acids," J. Chromatogr. A 1192, 173-179 (2008).

[4] AN Constable and WJ Brittain, "Characterization of polymer brushes in capillaries," Colloids Surf. A 308, 123-128 (2007).

[5] Jamil El-Ali, Peter K Sorger, and Klavs F Jensen, "Cells on chips," Nature 442, 403 (2006).

[6] Dongeun Huh, Wei Gu, Yoko Kamotani, James B Grotberg, and Shuichi Takayama, "Microfluidics for flow cytometric analysis of cells and particles," Physiol. Meas. 26, R73 (2005).

[7] Derek Stein, Frank HJ van der Heyden, Wiepke JA Koopmans, and Cees Dekker, "Pressure-driven transport of confined dna polymers in fluidic channels," Proc. Natl. Acad. Sci. U.S.A. 103, 15853-15858 (2006).

[8] Howard A Stone, Abraham D Stroock, and Armand Ajdari, "Engineering flows in small devices: microfluidics toward a lab-on-a-chip," Annu. Rev. Fluid Mech. 36, 381-411 (2004).

[9] J Goyon, A Colin, G Ovarlez, A Ajdari, and L Bocquet, "Spatial cooperativity in soft glassy flows," Nature 454, 84 (2008). 


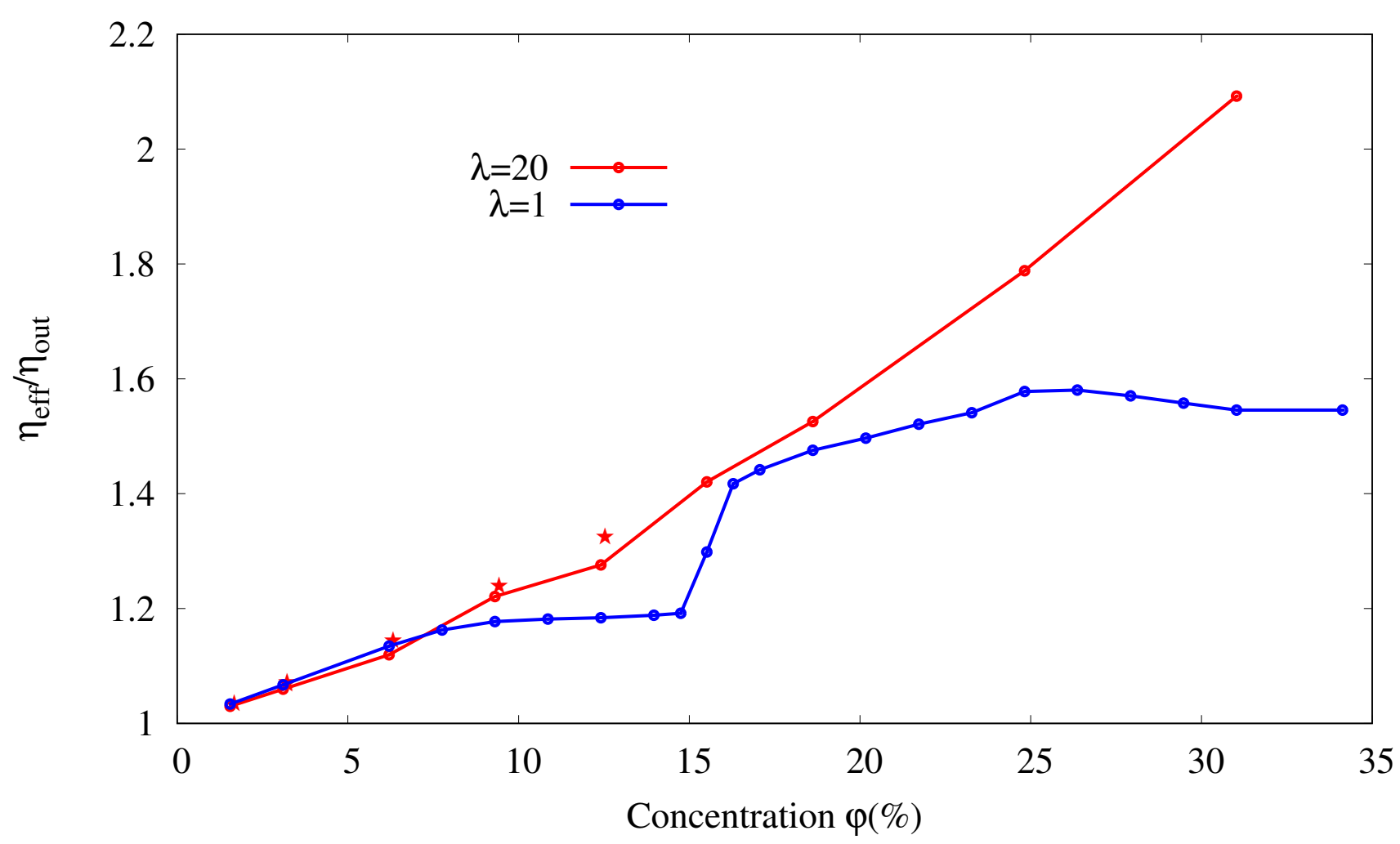

FIG. 6. (Color online) The evolution of the effective viscosity $\eta_{\text {eff }}$ as a function of concentration $\varphi$ for two values of $\lambda$. The filled circles (red curve) and the stars correspond to $\lambda=20$, a value which leads to different spatial configurations of the suspension which in turn give rise to different values of $\eta_{\text {eff }}$ (or $[\eta]$ ) as explained in the caption of Fig.5. The stars are the counterparts of the points $a^{\prime}-b^{\prime}$ and $b^{\prime \prime}$ in Fig.5. In the dilute regime the suspension of relatively rigid vesicles $(\lambda=20)$ may have a lower value of $\eta_{\text {eff }}$ than that of a suspension of soft vesicles $(\lambda=1)$. This is traced back to the existence of off-centered positions for $\lambda=20$ as discussed in the text. In the more concentrated regime, the suspension of relatively soft vesicles $(\lambda=1)$ has a much lower $\eta_{e f f}$ than that of a suspension of relatively rigid vesicles. This is traced back to the existence of ordered solutions with a double file configuration for $\lambda=1$ and disordered solutions for $\lambda=20$. See the text for more details.

[10] Roger Jäggi, Rainer D.and Sandoz and Carlo S. Effenhauser, "Microfluidic depletion of red blood cells from whole blood in high-aspect-ratio microchannels," Microfluidics and Nanofluidics 3, 47-53 (2007).

[11] Michael D Graham, "Fluid dynamics of dissolved polymer molecules in confined geometries," Annu. Rev. Fluid Mech. 43, 273-298 (2011).

[12] TV Starkey, "The laminar flow of streams of suspended particles," Br. J. Appl. Phys. 7, 52 (1956).

[13] G Segre and A Silberberg, "Radial particle displacements in poiseuille flow of suspensions," Nature 189, 209 (1961).

[14] Howard A Barnes, "A review of the slip (wall depletion) of polymer solutions, emulsions and particle suspensions in viscometers: its cause, character, and cure," J. Nonnewton Fluid Mech. 56, 221-251 (1995).

[15] Hongbo Ma and Michael D Graham, "Theory of shear-induced migration in dilute polymer solutions near solid boundaries," Phys. Fluids 17, 083103 (2005).

[16] Timothy W. Secomb, "Blood flow in the microcirculation," Annu. Rev. Fluid Mech. 49, 443-461 (2017).

[17] Pratik Pranay, Rafael G Henríquez-Rivera, and Michael D Graham, "Depletion layer formation in suspensions of elastic capsules in newtonian and viscoelastic fluids," Phys. Fluids 24, 061902 (2012).

[18] Hengdi Zhang, Zaiyi Shen, Brenna Hogan, Abdul I Barakat, and Chaouqi Misbah, "Atp release by red blood cells under flow: Model and simulations," Biophys. J. 115, 2218-2229 (2018).

[19] Robin Fåhræus and Torsten Lindqvist, "The viscosity of the blood in narrow capillary tubes," Am. J. Physiol. 96, 562-568 (1931).

[20] Philippe Peyla and Claude Verdier, "New confinement effects on the viscosity of suspensions," EPL 94, 44001 (2011).

[21] Yohan Davit and Philippe Peyla, "Intriguing viscosity effects in confined suspensions: A numerical study," EPL 83, 64001 (2008).

[22] Ashok S Sangani, Andreas Acrivos, and Philippe Peyla, "Roles of particle-wall and particle-particle interactions in highly confined suspensions of spherical particles being sheared at low reynolds numbers," Phys. Fluids 23, 083302 (2011).

[23] Marine Thiébaud, Zaiyi Shen, Jens Harting, and Chaouqi Misbah, "Prediction of anomalous blood viscosity in confined shear flow," Phys. Rev. Lett. 112, 238304 (2014). 
[24] Zaiyi Shen, Thomas M Fischer, Alexander Farutin, Petia M Vlahovska, Jens Harting, and Chaouqi Misbah, "Blood crystal: emergent order of red blood cells under wall-confined shear flow," Phys. Rev. Lett. 120, 268102 (2018).

[25] Walter Fornari, Luca Brandt, Pinaki Chaudhuri, Cyan Umbert Lopez, Dhrubaditya Mitra, and Francesco Picano, "Rheology of confined non-brownian suspensions," Phys. Rev. Lett. 116, 018301 (2016).

[26] Reinhard Lipowsky and Erich Sackmann, Structure and dynamics of membranes: I. from cells to vesicles/II. generic and specific interactions, Vol. 1 (Elsevier, 1995).

[27] Thierry Biben, Alexander Farutin, and Chaouqi Misbah, "Three-dimensional vesicles under shear flow: Numerical study of dynamics and phase diagram," Phys. Rev. E 83, 031921 (2011).

[28] Giovanni Ghigliotti, Thierry Biben, and Chaouqi Misbah, "Rheology of a dilute two-dimensional suspension of vesicles," J. Fluid Mech. 653, 489-518 (2010).

[29] A. Farutin and C. Misbah, "Analytical and numerical study of three main migration laws for vesicles under flow," Phys. Rev. Lett. 110, 108104 (2013).

[30] Badr Kaoui, George Biros, and Chaouqi Misbah, "Why do red blood cells have asymmetric shapes even in a symmetric flow?" Phys. Rev. Lett. 103, 188101 (2009).

[31] Abdessamad Nait-Ouhra, Achim Guckenberger, Alexander Farutin, Hamid Ez-Zahraouy, Abdelilah Benyoussef, Stephan Gekle, and Chaouqi Misbah, "Lateral vesicle migration in a bounded shear flow: Viscosity contrast leads to off-centered solutions," Phys. Rev. Fluids 3, 123601 (2018).

[32] Johannes Mauer, Simon Mendez, Luca Lanotte, Franck Nicoud, Manouk Abkarian, Gerhard Gompper, and Dmitry A Fedosov, "Flow-induced transitions of red blood cell shapes under shear," Phys. Rev. Lett. 121, 118103 (2018).

[33] Hengdi Zhang, Simulation of Blood Microcirculation and Its Coupling to Biochemical Signaling, Ph.D. thesis, University Grenoble-Alpes (2018).

[34] Gwennou Coupier, Badr Kaoui, Thomas Podgorski, and Chaouqi Misbah, "Noninertial lateral migration of vesicles in bounded poiseuille flow," Phys. Fluids 20, 111702 (2008).

[35] Piero Olla, "The lift on a tank-treading ellipsoidal cell in a shear flow," J. Phys. II France 7, 1533-1540 (1997).

[36] Isabelle Cantat and Chaouqi Misbah, "Lift force and dynamical unbinding of adhering vesicles under shear flow," Phys. Rev. Lett. 83, 880 (1999).

[37] Udo Seifert, "Hydrodynamic lift on bound vesicles," Phys. Rev. Lett. 83, 876 (1999).

[38] Sreejith Sukumaran and Udo Seifert, "Influence of shear flow on vesicles near a wall: a numerical study," Phys. Rev. E 64, $011916(2001)$.

[39] Piero Olla, "The behavior of closed inextensible membranes in linear and quadratic shear flows," Physica A 278, 87-106 (2000).

[40] Piero Olla, "The role of tank-treading motions in the transverse migration of a spheroidal vesicle in a shear flow," J. Phys. A: Math. Gene. 30, 317 (1997).

[41] Piero Olla, "Simplified model for red cell dynamics in small blood vessels," Phys. Rev. Lett. 82, 453 (1999).

[42] Badr Kaoui, GH Ristow, Isabelle Cantat, Chaouqi Misbah, and Walter Zimmermann, "Lateral migration of a twodimensional vesicle in unbounded poiseuille flow," Phys. Rev. E 77, 021903 (2008).

[43] Marine Thiébaud and Chaouqi Misbah, "Rheology of a vesicle suspension with finite concentration: A numerical study," Phys. Rev. E 88, 062707 (2013).

[44] Supplementary Information contains additional figures. (2019).

[45] Ou-Yang Zhong-Can and Wolfgang Helfrich, "Bending energy of vesicle membranes: General expressions for the first, second, and third variation of the shape energy and applications to spheres and cylinders," Phys. Rev. A 39, 5280 (1989).

[46] G. Batchelor, "The stress system in a suspension of force-free particles," J. Fluid Mech. 41, 545-570 (1970).

[47] G. Danker and C. Misbah, "Rheology of a dilute suspension of vesicles," Phys. Rev. Lett. 98, 088104 (2007).

[48] Hong Zhao and Eric SG Shaqfeh, "The dynamics of a non-dilute vesicle suspension in a simple shear flow," J. Fluid Mech. 725, 709-731 (2013).

[49] Abtin Rahimian, Shravan Kumar Veerapaneni, and George Biros, "Dynamic simulation of locally inextensible vesicles suspended in an arbitrary two-dimensional domain, a boundary integral method," J. Comp. Physics 229, 6466-6484 (2010).

[50] Victoria Vitkova, Maud-Alix Mader, Benoît Polack, Chaouqi Misbah, and Thomas Podgorski, "Micro-macro link in rheology of erythrocyte and vesicle suspensions," Biophys. J. 95, L33-L35 (2008).

[51] Gerrit Danker, Thierry Biben, Thomas Podgorski, Claude Verdier, and Chaouqi Misbah, "Dynamics and rheology of a dilute suspension of vesicles: Higher-order theory," Phys. Rev. E 76, 041905 (2007). 